\title{
¿Enseñamos morfología a través del diccionario? Reflexiones sobre la práctica docente en la Educación Secundaria
}

\section{Do we teach morphology through dictionaries? Lines of thought concerning teaching practice in Secondary Education}

\section{Lucía Marco Martínez}

Universidad Autónoma de Madrid marcomlucia@gmail.com

ORCID ID: https://orcid.org/0000-0002-4496-291X

DOI: $10.17398 / 1988-8430.35 .1 .175$

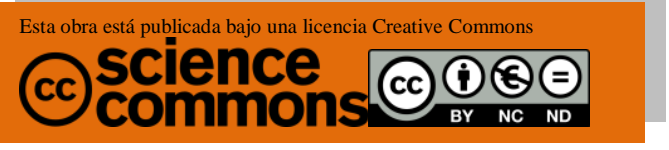

Fecha de recepción: 16/09/2021 Fecha de aceptación: 19/07/2021

Marco Martínez, L. (2021). ¿Enseñamos morfología a través del diccionario? Reflexiones sobre la práctica docente en la Educación Secundaria. Tejuelo, 35(1), 175-208.

Doi: https://doi.org/10.17398/1988-8430.35.1.175 
Resumen: Este artículo investiga el uso del diccionario para explicar morfología en la Educación Secundaria. Por lo tanto, trabajaremos en torno a dos variables: el uso del diccionario y el tratamiento de la morfología en un contexto determinado. Se desarrolla un estudio preliminar sobre la práctica docente a través de un cuestionario cumplimentado por treinta profesores de Lengua Castellana y Literatura. Los resultados ilustran cuál es el lugar que ocupa el diccionario en la educación actual y permite dar cuenta de los puntos de mejora. Asimismo, se presentan unas líneas didácticas para integrar el diccionario en el aprendizaje de la morfología.

Palabras clave: lexicografía didáctica; diccionario; morfología; morfología léxica; didáctica.
Abstract: This paper examines the use of dictionaries for teaching morphology in Secondary Education. Therefore, we are going to work on the use of dictionaries and morphology in a specific context. This preliminary study about teaching practice has been developed through a form completed by thirty teachers. The results show the dictionary use in a high school level and this takes full account of the areas that can be improved. Likewise, some didactic guidance is presented to help teachers in implementing the dictionary to teach morphology.

Keywords: learners' lexicography; dictionary; morphology; lexical morphology; teaching. 


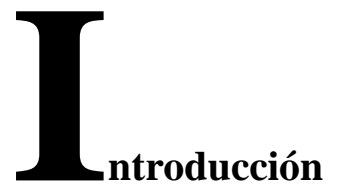

A lo largo del curso escolar, el profesorado de Educación Secundaria Obligatoria (ESO) trabaja con unas herramientas didácticas concretas, como los manuales, según la aceptación de las directrices marcadas tanto por el centro escolar como por la ley educativa vigente. En esta fase, el docente valora los aspectos favorables y desfavorables de los libros de texto y asume que no existe una obra perfecta que se adapte totalmente a las necesidades específicas de su aula. Por ello, intenta combinar la línea educativa del centro con su metodología a través de la preparación de diversas tareas que cubran las carencias del alumnado.

Además de los manuales, el profesorado cuenta con varios recursos en los que puede apoyar sus explicaciones: desde las actividades propuestas por los grupos editoriales y el uso de las Tecnologías de Información y Comunicación (las TIC), hasta el empleo de materiales ya conocidos como los diccionarios, las gramáticas o los libros de lectura. El profesor selecciona los materiales que se van a usar 
en el curso y determina la metodología adoptada en la explicación de los contenidos. Es en esta etapa cuando el profesor se plantea el uso del diccionario en la clase.

Existen varios estudios que ponen de manifiesto los beneficios didácticos de las obras lexicográficas (Maldonado González, 1998; Martín García, 1999, 2015; Azorín Fernández, 2000; Alvar Ezquerra, 2003; Rodríguez Márquez, 2017, entre otros). Sin embargo, el día a día nos muestra una realidad distinta donde esta herramienta didáctica no está tan presente para abordar las cuestiones gramaticales (Prado Aragonés, 2005 y Rodríguez Marquez, 2017, p.155). A partir de esta observación, hemos decidido iniciar un estudio sobre el uso del diccionario monolingüe a la hora de explicar cuestiones morfológicas en la ESO. Con esta reflexión sobre la práctica docente, se pretende mostrar a los profesores la utilidad de este material complementario para enseñar morfología.

El artículo está dividido en tres apartados. En el primero, revisaremos algunos estudios previos para mostrar los beneficios que aporta el diccionario en la enseñanza de la morfología en la ESO. Hemos elegido esta etapa educativa, ya que la relevancia del estudio de la morfología se une a la enseñanza de las herramientas de información (los diccionarios, las bibliotecas y las TIC, entre otros) en una fase en la que se fomenta el aprendizaje autónomo del alumno a través de metodologías centradas en el estudiante. Por todo ello, creemos que es el momento idóneo para estudiar la relación entre la morfología y el diccionario. En la segunda sección, presentaremos una encuesta realizada a los docentes y analizaremos sus reflexiones sobre el uso de las obras lexicográficas para explicar cuestiones morfológicas en este ciclo académico. Finalmente, plantearemos una serie de pautas orientativas para enseñar morfología a través del diccionario.

\section{1.- La morfología y el diccionario en la Educación Secundaria}

Como sabemos, la morfología es un campo de la gramática que tiene como objeto de estudio la estructura interna de las palabras, lo que conlleva comprender los elementos morfológicos, los procesos de 
combinación o las propias categorías gramaticales (RAE y ASALE, 2009). La unidad de análisis del nivel morfológico es el morfema, la unidad gramatical mínima con significado. Según las propiedades de los componentes y de los procesos, el análisis morfológico se divide en dos ramas: la morfología flexiva y la morfología léxica. La primera se ocupa de los morfemas que añaden contenidos gramaticales, como el género, el número, la persona, el tiempo o el modo, imprescindibles para expresar la concordancia. Por otro lado, la morfología léxica estudia los procesos morfológicos de formación de palabras mediante la adjunción de morfemas que aportan información léxica a la palabra resultante. Si bien un modelo de la enseñanza del léxico basado en la morfología flexiva ayuda a entender la relación de concordancia de las unidades léxicas con otros términos (Martínez Fraile, 2015 y Saragossà Alba, 2015), un modelo del aprendizaje del léxico centrado en la morfología léxica permitirá la mejor comprensión de las palabras derivadas y compuestas, su relación con otras unidades léxicas y su producción para hacer discursos más precisos.

La morfología es una de las disciplinas más relevantes de la Lingüística en la ESO, ya que, además de ampliar el vocabulario del alumnado y de facilitar el reconocimiento de las categorías gramaticales y la estructura de las palabras, es la base para la construcción de las estructuras sintácticas. En algunos estudios como el de Nation (1990), se pone de relieve que el conocimiento de una palabra abarca información sobre distintos aspectos: su pronunciación y ortografía, los morfemas que la constituyen, su combinación con otras palabras en la sintaxis, la frecuencia de uso, su inclusión en contextos pragmáticos para adecuarla a una situación comunicativa concreta y sus acepciones y usos figurados. De esta manera, aprender una palabra implica conocer conceptos y estrategias para utilizar el término de forma precisa y adecuada en textos orales y escritos. Como afirma Matilla Ocaña (2006, p. 223), la adquisición de vocabulario (su significado y su buen uso) es la base para una buena comprensión de textos y para una correcta expresión oral y escrita.

Asimismo, como ya han señalado algunos autores (por ejemplo, Nippold, 2006 y Levie, Ben-Zvi y Ravid, 2016), el análisis morfológico 
se presenta como una de las habilidades lingüísticas principales para la adquisición del léxico. Para ello, el estudiante debe identificar los morfemas constituyentes de una palabra, conocer su significado, definir cómo los afijos modifican el significado de la raíz y combinar las piezas léxicas para comprender el significado del término nuevo (Larsen y Nippold, 2007). También la conciencia morfológica conlleva el reconocimiento de los morfemas y sus funciones gramaticales (Kieffer y Lesaux, 2012 y Martín García, 2015), así como el establecimiento de las relaciones semánticas entre las diversas formas morfológicas que permiten la creación de una red semántica organizada, donde las palabras se asocian mediante una jerarquía conceptual (Martín García, 2015 y Martín Vegas, 2018). De esta forma, el estudiante aprenderá nuevas palabras y podrá usar su conocimiento morfológico para comprender los términos desconocidos en contexto.

Respecto a las obras lexicográficas, según muestran algunos autores (Alvar Ezquerra, 1993, 2003; Azorín Fernández, 2000; Martín García, 1999, 2005; y Martín Vegas, 2018; entre otros), el diccionario monolingüe es un material con una función didáctica clara, ya que nos explica las características intrínsecas de los términos (la pronunciación, las relaciones de significado, la morfología, etc.) en un contexto determinado, con el objetivo de que el usuario sea capaz tanto de comprender la palabra como de usarla adecuadamente. Por este motivo, observamos que el diccionario es una herramienta de apoyo útil para describir el funcionamiento de las palabras, por lo cual debe estar presente en las clases de Lengua Castellana y Literatura. Sin embargo, cabe preguntarse, por un lado, si los profesores utilizan este recurso como apoyo en sus explicaciones y, por otro, cuáles son las dificultades del profesorado y del alumnado en el uso de los diccionarios en las clases de Lengua Castellana y Literatura. Cuestiones semejantes han sido destacadas en distintos trabajos (Hernández Hernández, 1989; Azorín Fernández, 2000; Sánchez Muñoz, 2003; Rodríguez Márquez, 2017).

A partir de la década de los noventa, en España se produce un interés editorial por publicar diccionarios destinados a estudiantes con una clara finalidad didáctica (Hernández Hernández, 1996 y 1998), 
interés que se ha mantenido hasta nuestros días. Sin embargo, la realidad muestra que el desconocimiento de los docentes sobre el uso de este recurso lexicográfico hace que se infrautilice en los centros escolares (Prado Aragonés, 2005). Por tanto, es de esperar que, si el empleo del diccionario tiene poca cabida en las clases de Lengua Castellana y Literatura durante esta etapa, su presencia a la hora de explicar morfología también será escasa.

Tanto la morfología como los diccionarios están incluidos en las leyes educativas vigentes. En el currículo, los contenidos morfológicos se centran en la observación reflexiva de la palabra, con el objetivo de que el estudiante haga un uso correcto de la misma en un contexto comunicativo determinado. A lo largo del primer ciclo de la ESO, según se especifica en el documento ${ }^{1}$, se tratan los elementos constitutivos de la palabra y los diferentes procedimientos que los combinan. Asimismo, durante el último curso, los conocimientos que adquiere el estudiante son más específicos y se plasman en la reflexión sobre el uso de las categorías gramaticales, en el estudio de los prefijos y sufijos y en su capacidad para la formación de nuevos términos. En cuanto al diccionario, se presenta como una obra que recoge palabras y que resuelve dudas sobre el uso correcto de la lengua, al igual que otras fuentes de consulta en papel o en línea (gramáticas, páginas web, corpus o enciclopedias). De este modo, los diccionarios tienen una doble finalidad. Por un lado, el estudiante puede conocer y comprender una palabra y, por otro, puede aprender a usarla correctamente en sus producciones orales y escritas. Asimismo, durante los dos ciclos de la ESO, el currículo incide en el uso progresivo de diversas obras lexicográficas con el objetivo de que el alumnado sea capaz de utilizarlas de manera autónoma y eficaz.

El conocimiento de la morfología y el uso del diccionario están tratados en las Competencias Clave (Ministerio de Educación y Formación Profesional, 2015) en el aula de Lengua Castellana y Literatura. En primer lugar, con el uso del diccionario se fomenta el desarrollo de la competencia lingüística, gracias a la información

${ }^{1}$ Nos referimos al Real Decreto 1105/2014 y al Decreto 48/2015 de la Comunidad de Madrid, que regulan la enseñanza de la Lengua y Literatura castellanas. 
codificada que incluyen los diccionarios. Para que esta información sea relevante y favorezca una comunicación eficiente y natural en español en cualquier situación comunicativa, el estudiante tendrá que saber interpretar los datos incluidos en las obras lexicográficas. Como ejemplo mostramos el artículo lexicográfico del verbo pensar, según aparece codificado en el Diccionario de aprendizaje del español como lengua extranjera (DAELE), un prototipo de diccionario desarrollado para español como lengua extranjera. Dado el carácter didáctico de este diccionario y su formato en línea, consideramos que puede ser de utilidad igualmente para los estudiantes de secundaria.

\section{Figura 1}

Entrada de pensar en el DAELE

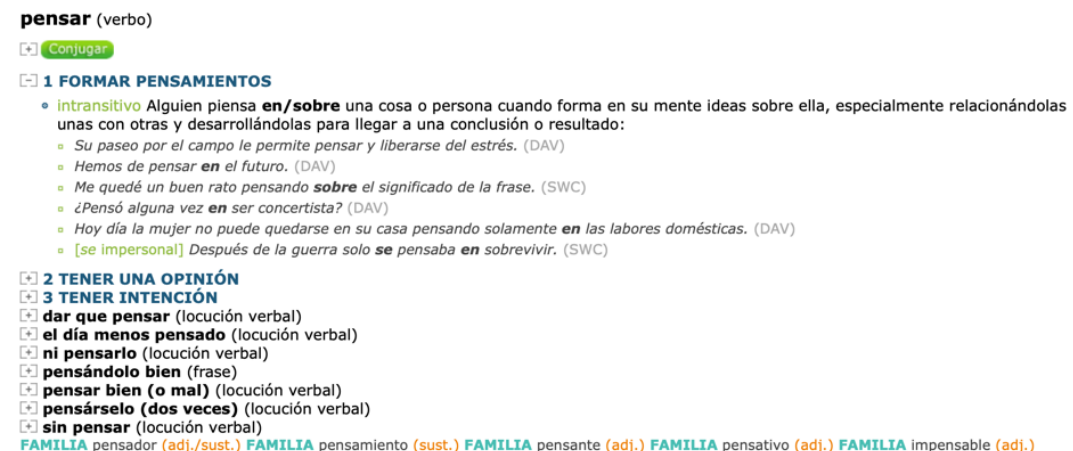

Fuente: DAELE, s.f.

En este caso, el usuario puede comprobar la ortografía del verbo a partir del lema y conocer su significado en la definición. Además, la microestructura incluye información como la categoría y la subcategoría gramatical, a través de marcas explícitas en la metalengua (verbo e intransitivo). También muestra la marcación de los argumentos verbales en la definición mediante pronombres indefinidos (alguien o algo) o sustantivos generales (cosa o persona, entre otros), así como las alternancias del verbo (se impersonal). Asimismo, el diccionario incluye ejemplos para conocer el uso del término en un contexto y permite acceder a la conjugación del verbo pensar a través del botón "Conjugar". Por último, las palabras contenidas en el apartado "FAMILIA" permiten establecer relaciones entre palabras de distinta categoría gramatical dentro de una familia léxica. Datos semejantes a 
los anteriores facilitan la comprensión de una palabra para que pueda ser usada en un contexto determinado.

Un diccionario como el DAELE, con una finalidad claramente didáctica que fomenta la función codificadora y descodificadora, permite que el usuario adquiera vocabulario. Por un lado, la función descodificadora está ligada a la comprensión de enunciados, en la medida en que el diccionario aporta el significado de la palabra que se busca. Esta función tradicional es el fundamento de un diccionario pasivo $\mathrm{y}$, en muchas ocasiones, demuestra el uso común que hacemos de los diccionarios en el aula 2 . Por otro lado, la función codificadora aboga por exponer cómo funciona la palabra en un contexto, con el objetivo de comprender y producir textos y oraciones con sentido. Esta función es la base de un diccionario activo, ya que permite que el usuario pueda reflexionar sobre el término a partir de los conocimientos lingüísticos previos.

En segundo lugar, el diccionario contribuye también al conocimiento cultural a través del mundo que se plasma en la obra y a partir de las referencias hechas a un contexto cultural concreto (Dubois y Dubois, 1971). Este hecho tiene especial importancia en los neologismos y en los préstamos que se toman de otras lenguas, ya que con la incorporación de la forma se adopta también una noción cultural asociada a la palabra. Además, en los diccionarios se consigna la procedencia de los préstamos.

En tercer lugar, al recibir formación sobre lexicografía, los estudiantes pueden ser capaces de seleccionar el diccionario que mejor se ajuste a sus necesidades para que, posteriormente, puedan usarlo de manera autónoma. En otras palabras, el alumno debe saber que hay un diccionario específico para cada necesidad (diccionario monolingüe, bilingüe, de dudas, de sinónimos y antónimos, etc.) y debe ser capaz de elegir el más adecuado. Además, si el estudiante está familiarizado con la estructura y la codificación del diccionario, puede encontrar la

\footnotetext{
${ }^{2}$ Como expone Azorín (2000, p.34), las tres primeras finalidades de una obra lexicográfica en el contexto educativo son el conocimiento de significado de las unidades léxicas, la verificación ortográfica y la búsqueda de sinónimos y antónimos.
} 
información que busca en un determinado momento, de modo que el diccionario se convierta, realmente, en un instrumento de aprendizaje. En este proceso, el estudiante está desarrollando la competencia de aprender a aprender. Asimismo, gracias al uso de diversos materiales (los diccionarios, los corpus, las bases de datos o los textos paralelos) y a la inclusión de algunas estrategias de aprendizaje (cognitiva, metacognitiva y memorística) se desarrollan la autonomía y la iniciativa del alumnado (Hernández Hernández, 1991, p. 195; Sánchez García, 2015).

La formación sobre los diccionarios es fundamental también para saber discriminar los diccionarios en línea, ya que actualmente la oferta de este tipo de diccionarios es amplia y no todos ofrecen la misma calidad (De Schryver, 2003). La evolución tecnológica que ha experimentado la lexicografía ha permitido que los diccionarios incluyan más datos sobre las palabras y que la información se presente de una forma más intuitiva. Por ejemplo, en el diccionario DUEACLAVE (Figura 2), al buscar el término surfear, el estudiante puede usar un hipervínculo en el término surf (Figura 3), donde encontrará otros datos relacionados con el lema. De esta manera, el estudiante creará relaciones entre surfear y surf y entenderá que pertenecen a la misma familia léxica. Las incuestionables ventajas que proporcionan estos diccionarios deben ser conocidas por los estudiantes con el fin de que las usen de forma adecuada.

\section{Figura 2}

Entrada de surfear en el DUEA-CLAVE

\section{surfear surfe-ar}

$$
\text { v. }
$$

1 Practicar surf: Hemos surfeado durante toda la mañana porque había muy buenas olas.

2 Navegar por internet sin buscar nada concreto: Cada vez que surfeo en internet me encuentro cosas más raras.

IMORFOLOGA Verbo reg. $\rightarrow$.

Fuente: DUEA-CLAVE, s.f. 
Figura 3

Entrada de surf en el DUEA-CLAVE

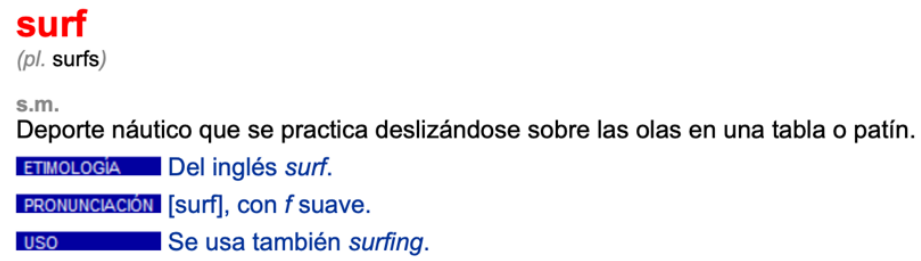

Fuente: DUEA-CLAVE, s.f.

Dado que las leyes educativas vigentes contemplan la compresión integral de la palabra a través del diccionario, los profesores deberían enseñar a usar esta herramienta (Cassany Comas, Luna Sanjuan y Sanz Pinyol, 1994). De forma más específica, varios autores defienden el uso del diccionario para desarrollar la competencia morfológica (Martínez Ezquerro, 2001; Graves, 2006; Martín Vegas, 2018; Hess Zimmermann, 2019). Así, Hess Zimmermann (2019) aboga por el uso del diccionario en todos los ámbitos de la Lingüística (semántica, sintaxis, morfología, fonética) para que el estudiante comprenda el término en su totalidad y sepa utilizarlo en el contexto adecuado. La autora, a través de su estudio, anima a conocer la estructura de la palabra, los patrones de composición y su categoría gramatical a través del uso y de la creación de un diccionario. También Graves (2006) y Martín Vegas (2018) subrayan que la descomposición morfológica de las palabras y el diccionario son dos elementos clave para que el alumnado incluya nuevos términos en su vocabulario de manera eficaz. La obra lexicográfica ayuda a los estudiantes a comprender las relaciones entre familias y permite que estos amplíen las conexiones que se generan en el lexicón mental.

Una vez que hemos mostrado la importancia del diccionario para aprender vocabulario y para desarrollar el conocimiento morfológico, es preciso analizar el uso del diccionario en el aula para explicar cuestiones morfológicas en la ESO. 


\section{2.- El diccionario para explicar morfología en el aula 2.1.- Metodología}

Si bien las leyes educativas vigentes incluyen en sus currículos tanto el estudio de la morfología como el uso del diccionario y se recomienda su incorporación en las clases de Lengua Castellana y Literatura, cabe preguntarnos por qué los docentes utilizan tan poco este recurso para explicar gramática. Con el objetivo de conocer la actitud del profesorado con estas obras para explicar morfología en la ESO, hemos elaborado un cuestionario sobre la frecuencia de uso y el aprovechamiento del diccionario. En el planteamiento de la encuesta, hemos tenido en cuenta varias variables, como el ciclo o nivel en el que imparten docencia o el conocimiento sobre lexicografía de los encuestados, $\mathrm{y}$ hemos añadido preguntas abiertas para que el profesorado pueda expresar su opinión libremente sobre algunos temas.

El cuestionario sobre la práctica del profesorado consta de veintiuna preguntas. Sin embargo, nos centramos en las más relevantes para la investigación. El esquema de la encuesta se ha estructurado de la siguiente forma. El primer apartado está destinado a la identificación de los docentes (el sexo, la edad, el nivel de estudios y el conocimiento lexicográfico). La segunda sección recoge los datos sobre el centro educativo donde trabajan los encuestados (la titularidad, la localidad y el nivel de enseñanza en el que imparten clase). El tercer bloque se centra en el uso del diccionario en la ESO (frecuencia de uso y tipo de diccionario utilizado, entre otras cuestiones). Por último, el cuarto apartado hace referencia a la utilización del diccionario para enseñar morfología (cómo se integra el material en el aula y qué contenidos morfológicos se explican).

El formulario fue completado durante el curso escolar 20192020 por treinta informantes de características heterogéneas que accedieron a él de manera telemática a través de Google Drive, plataforma en la que fue creado el cuestionario. Como veremos a continuación, tanto los datos sobre las características personales de los encuestados como aquellos relativos a la práctica docente nos acercan a 
la situación actual del uso del diccionario para enseñar morfología en el aula.

\section{2.- La comunidad educativa y el diccionario: resultados obtenidos}

El primer apartado de la encuesta "Composición de la muestra del profesorado encuestado" nos permitió conocer los datos personales de los docentes, como puede apreciarse en la siguiente tabla:

\section{Tabla 1}

Composición de la muestra del profesorado encuestado

\begin{tabular}{lc}
\hline Edad & $\begin{array}{c}\mathbf{N}^{\mathbf{0}} \mathbf{d e} \\
\text { informantes }\end{array}$ \\
\hline Menos de 30 & 7 \\
$30-40$ & 8 \\
$40-50$ & 10 \\
Más de 50 & 5 \\
\hline
\end{tabular}

\section{Género}

$\mathrm{N}^{\mathbf{0}}$ de

informantes

Hombre 7

Mujer 23

\begin{tabular}{|c|c|}
\hline Nivel de estudios & $\begin{array}{c}\mathrm{N}^{\circ} \text { de } \\
\text { informantes }\end{array}$ \\
\hline Grado o Licenciatura & 16 \\
\hline Máster & 11 \\
\hline Doctorado & 3 \\
\hline Estudios en lexicografía & $\begin{array}{c}\mathbf{N}^{\circ} \text { de } \\
\text { informantes }\end{array}$ \\
\hline Sí & 10 \\
\hline No & 20 \\
\hline Lugar de trabajo & $\begin{array}{c}\mathrm{N}^{0} \mathrm{de} \\
\text { informantes } \\
\end{array}$ \\
\hline Centro de Educación Secundaria público & 12 \\
\hline Centro de Educación Secundaria concertado & 15 \\
\hline Centro de Educación Secundaria privado & 3 \\
\hline
\end{tabular}

Fuente: elaboración propia 
La formación es una cuestión relevante en la docencia para actualizar contenidos, ya que el profesorado debe tener una base lingüística sólida para poder transmitir los conocimientos a los estudiantes. Si bien es cierto que todos los participantes han obtenido el título de grado o de licenciatura para poder ejercer como profesores de Lengua Castellana y Literatura, solo catorce docentes han continuado su formación universitaria con estudios superiores de máster o de doctorado. Sin embargo, el nivel de estudios no es, en absoluto, un indicativo del grado de familiaridad de los docentes con el diccionario. Con el objetivo de conocer esta cuestión, incluimos una pregunta relativa a la formación lexicográfica y comprobamos que solo diez participantes han realizado cursos específicos en este campo.

Además de una formación específica, los conocimientos sobre lexicografía también se pueden adquirir de manera autodidacta a través de la práctica con los diccionarios. Por ello, es relevante conocer con qué frecuencia acuden los encuestados al diccionario para resolver cuestiones lingüísticas. Para la pregunta “¿Utiliza el diccionario habitualmente?", obtenemos las siguientes respuestas:

\section{Tabla 2}

Pregunta 1

\begin{tabular}{lcc}
\hline ¿Utiliza el diccionario habitualmente? & \% & N. $^{\mathbf{0}}$ de informantes \\
\hline Sí & 46,7 & 14 \\
No & 16,7 & 5 \\
A veces & 36,7 & 11 \\
\hline
\end{tabular}

Fuente: elaboración propia

Como se observa en la tabla, el 46,7\% de los profesores utiliza el diccionario de manera habitual, un $36,7 \%$ lo consulta en alguna ocasión y solo un $16,7 \%$ de los encuestados confiesa no usarlo.

Respecto al contexto educativo actual, debemos subrayar que los encuestados trabajan en un centro educativo supeditado a la ley educativa vigente y a las concreciones curriculares de las Comunidades Autónomas, ámbitos legales en los que el uso del diccionario es un objetivo claro. Además, es imprescindible conocer si en el aula los 
docentes disponen de obras lexicográficas. Como veremos en la siguiente tabla, en las clases de Lengua Castellana y Literatura, la forma de consulta del diccionario difiere de unos docentes a otros.

\section{Tabla 3}

Pregunta 2

\begin{tabular}{|c|c|c|}
\hline $\begin{array}{l}\text { ¿Los estudiantes tienen acceso a un } \\
\text { diccionario en papel o en línea en clase de } \\
\text { Lengua? }\end{array}$ & $\%$ & N. ${ }^{\circ}$ de informantes \\
\hline Sí, en papel & 16,7 & 5 \\
\hline Sí, en línea & 33,3 & 10 \\
\hline Sí, en papel y en línea & 46,7 & 14 \\
\hline No & 3,3 & 1 \\
\hline
\end{tabular}

Fuente: elaboración propia

La mayoría de los docentes tiende a usar diccionarios en papel y en línea, como refleja el 46,7\% de los encuestados, seguido de un $33,3 \%$ de docentes que los consulta en plataformas en línea o en aplicaciones y de un $16,7 \%$ de profesores que dispone de diccionarios solo en papel. Frente a estos datos, encontramos que un 3,3\% de los participantes no puede acceder directamente a un diccionario en papel o en línea dentro del aula.

Según muestran los datos anteriores, en clase de Lengua Castellana y Literatura los alumnos disponen de material lexicográfico en papel y en línea. A partir de esta evidencia, es preciso conocer si los estudiantes usan los diccionarios en clase. Como reflejan los datos de la siguiente tabla, el empleo del material lexicográfico en la ESO es significativo, ya que un $76,6 \%$ de los encuestados confirma su utilización. Dentro de este grupo, mientras que un 56,7\% declara usar el diccionario y explicar a los estudiantes cómo deben utilizarlo, un $20 \%$ justifica que lo maneja, pero no facilita ninguna instrucción a su alumnado. Frente a estos datos positivos, un 23,3\% decide no introducir este material en el aula. 
Tabla 4

Pregunta 3

\begin{tabular}{lcc}
\hline ¿Usa el diccionario en clase con el alumnado? & \% & N. $^{\mathbf{0}}$ de informantes \\
\hline Sí y les explico cómo tienen que usarlo & 56,7 & 17 \\
Sí, pero no les explico cómo tienen que usarlo & 20 & 6 \\
No & 23,3 & 7 \\
\hline
\end{tabular}

Fuente: elaboración propia

La pregunta anterior hace referencia directa a la práctica docente y nos plantea dos cuestiones interesantes: la metodología y la frecuencia de uso. Por un lado, la mayoría del profesorado cree conveniente guiar al estudiante en su proceso de aprendizaje y afirma incorporar en sus clases el uso dirigido o pautado del diccionario. Por otro lado, aunque observamos que el profesorado recurre al uso del diccionario en el aula, la utilización de este tipo de obras no es un indicador claro del grado de familiaridad de los estudiantes con el material (Azorín Fernández, 2000). A pesar de su indiscutible presencia en la mayoría de los casos, las obras lexicográficas son un instrumento de consulta opcional $\mathrm{y}$, al contrario de otros materiales como el libro de texto, la frecuencia de consulta varía según el encuestado. Los resultados de la pregunta “¿En qué momento utilizan el diccionario en el aula?" muestran que el alumnado sí usa el diccionario. Así, mientras que un 43,3\% de informantes siempre tiene el material a mano para resolver dudas, un $30 \%$ afirma que los estudiantes recurren a él cuando el docente se lo indica y un $26,7 \%$ lo usa muy poco debido a la falta de tiempo.

\section{Tabla 5}

Pregunta 4

\begin{tabular}{lcc}
\hline $\begin{array}{l}\text { ¿En qué momento utilizan el diccionario en el } \\
\text { aula? }\end{array}$ & $\%$ & $\mathbf{N .}^{\mathbf{0}}$ de informantes \\
\hline $\begin{array}{l}\text { Lo tienen siempre a mano para resolver } \\
\text { cualquier duda que les surja }\end{array}$ & 43,3 & 13 \\
$\begin{array}{l}\text { Solo lo usan cuando el profesor recomienda su } \\
\text { utilización }\end{array}$ & 30 & 9 \\
$\begin{array}{l}\text { Lo usan muy poco, ya que el tiempo es muy } \\
\text { limitado }\end{array}$ & 26,7 & 8 \\
Nunca. Es un material prescindible & 0 & 0 \\
\hline
\end{tabular}

Fuente: elaboración propia 
Ante los cuatro posibles escenarios planteados sobre la frecuencia de uso del diccionario, solo nos vamos a centrar en los tres primeros, ya que todos los profesores han afirmado que, en cierto modo, el material es imprescindible en la clase de Lengua Castellana y Literatura. El primer escenario indica un grado de integración alto en los hábitos del estudiante, ya que es capaz de usarlo de manera autónoma para resolver las dudas que tenga en cualquier ocasión. En el segundo escenario, el alumno aún no ha conseguido interiorizar la costumbre de acudir a la obra lexicográfica cuando tiene alguna duda. Al no tener autonomía, el estudiante solo recurre al material cuando el profesor se lo sugiere. Por último, el tercer escenario señala que el uso del diccionario es muy escaso, porque está condicionado al tiempo. Es decir, el estudiante no ha estado expuesto a la utilización de material lexicográfico en el aula y, por ello, no ha asumido esta tarea como algo rutinario.

Asimismo, nos interesamos por las recomendaciones de los docentes en el uso del diccionario en el aula, así como por el tipo de diccionario utilizado. Como vemos en la siguiente tabla, mientras que un $50 \%$ de los profesores deciden dar algún consejo que facilite su elección, el otro $50 \%$ se opone a esta recomendación.

\section{Tabla 6}

Pregunta 5

\begin{tabular}{lcc}
\hline $\begin{array}{l}\text { ¿Recomienda a sus estudiantes el uso de algún } \\
\text { diccionario en concreto? }\end{array}$ & $\%$ & $\mathbf{N .}^{\boldsymbol{0}}$ de informantes \\
\hline Sí & 50 & 15 \\
No & 50 & 15 \\
\hline
\end{tabular}

Fuente: elaboración propia

Los profesores que aconsejan el uso de una obra lexicográfica respondieron en una pregunta abierta que se decantan por el Diccionario de la Lengua Española (DLE) y por Wordrefence (WR). Es decir, un 53,33\% recomienda la obra de la Academia y un 26,66\% WR. Igualmente, tenemos que puntualizar que, después del profesorado, es el centro educativo, el ámbito familiar y, finalmente, el propio estudiante los que eligen la obra. Esto indica que la enseñanza 
del empleo del diccionario no está limitada únicamente al contexto escolar.

Además de estudiar el uso del diccionario de manera generalizada en la asignatura de Lengua Castellana y Literatura, hemos querido conocer qué piensan los profesores sobre la relación entre la morfología y los diccionarios. Antes de abordar este tema, consideramos fundamental la opinión que tienen los docentes acerca del uso del diccionario para explicar cuestiones morfológicas. Para ello, los encuestados respondieron a la pregunta "¿Piensa que el uso del diccionario facilita la adquisición del conocimiento morfológico?" y se obtuvieron las siguientes respuestas:

\section{Tabla 7}

Pregunta 6

\begin{tabular}{lcc}
\hline $\begin{array}{l}\text { ¿Piensa que el uso del diccionario facilita la } \\
\text { adquisición de conocimiento morfológico? }\end{array}$ & $\%$ & $\mathbf{N .}^{\mathbf{0}}$ de informantes \\
\hline Sí & 63,3 & 19 \\
No & 36,7 & 11 \\
\hline
\end{tabular}

Fuente: elaboración propia

Mientras que la mayoría de los profesores considera que el uso del diccionario favorece el proceso de aprendizaje de los conocimientos morfológicos, el 36,7\% restante opina que no. En una pregunta con respuesta abierta, los primeros piensan que el uso del diccionario es de gran utilidad para tratar las categorías gramaticales, el verbo (la irregularidad verbal y su morfología flexiva), el reconocimiento y la comprensión de los morfemas derivativos, la raíz léxica, los procedimientos de formación de las palabras y la neología.

Si bien es cierto que cada vez son más los profesores que afirman que el diccionario facilita la adquisición del conocimiento morfológico, los datos sobre la relación entre el uso de material lexicográfico y la morfología son menos alentadores. La encuesta muestra que aún no se ha interiorizado el uso de obras lexicográficas para tratar cuestiones morfológicas, como puede apreciarse en la tabla siguiente: 
Tabla 8

Pregunta 7

\begin{tabular}{lcc}
\hline ¿Usa el diccionario para enseñar morfología? & \% & N. $^{\mathbf{0}}$ de informantes \\
\hline Sí & 33,3 & 10 \\
No & 66,7 & 20 \\
\hline
\end{tabular}

Fuente: elaboración propia

Ante la pregunta "UUsa el diccionario para enseñar morfología?", solo un 33,33\% de los participantes opta por incorporar el diccionario para explicar cuestiones morfológicas. Estos docentes coinciden con aquellos que opinan que el diccionario facilita la adquisición de la morfología en la mayoría de los conceptos lingüísticos, entendiendo dentro de la morfología tanto la flexiva como la léxica.

Además de los contenidos que se incluyen, es relevante conocer cómo el docente integra el diccionario durante las sesiones. De esta manera, podemos averiguar si está familiarizado con el diccionario como herramienta para enseñar morfología. Mientras que un 56,66\% ha confesado que no lo usa en este contexto, un 43,32\% de los profesores afirma introducirlo en sus clases. Dentro de este último porcentaje, nos encontramos ante un 36,66\% de profesores que elabora sus propias actividades para aprovechar la finalidad didáctica del diccionario y solo un $6,66 \%$ de docentes se decanta por hacer las actividades que propone el libro de texto. A continuación, se presentan los datos:

\section{Tabla 9}

Pregunta 8

\begin{tabular}{lcc}
\hline ¿Cómo integra el diccionario en su clase? & $\%$ & N. ${ }^{\circ}$ de informantes \\
\hline $\begin{array}{l}\text { Hago las actividades del libro de texto que } \\
\text { indican el uso del diccionario para explicar }\end{array}$ & 6,66 & 2 \\
morfología & & 11 \\
$\begin{array}{l}\text { Elaboro actividades relacionadas con el manejo } \\
\text { del diccionario y la morfología }\end{array}$ & 36,66 & 17 \\
No uso el diccionario para explicar morfología & 56,66 & 17 \\
\hline
\end{tabular}

Fuente: elaboración propia

Una vez presentados los datos de la encuesta realizada a los docentes de secundaria, es el momento de analizar los resultados. 


\section{3.- Análisis de los resultados}

Según muestran los datos de la encuesta, los docentes valoran positivamente el diccionario y lo consideran como una obra de consulta usual tanto en la vida privada como en la vida profesional, a pesar de que hayan recibido poca formación sobre lexicografía a lo largo de su carrera docente. Además de esta buena predisposición, en casi todas las aulas se puede consultar un diccionario. El acceso generalizado a este recurso se ha producido gracias al desarrollo de internet, ya que hasta hace unos años la consulta de diccionarios en papel era lo habitual (De Schryver, 2003). En la actualidad, casi todos los centros educativos están preparados tecnológicamente para que se pueda hacer una búsqueda en un diccionario en línea. Además, el propio alumno puede acceder también a estas obras a través de su dispositivo móvil.

Las dos obras lexicográficas más utilizadas en el aula son el DLE y WR. Aunque los dos son diccionarios generales en línea destinados a un hablante cuya lengua materna es el español, como obras lexicográficas presentan varias diferencias. El DLE es el resultado de un proyecto lexicográfico llevado a cabo por la Real Academia de la Lengua Española y la Asociación de Academias de la Lengua Española y se actualiza al menos una vez al año. WR, por su parte, es una plataforma polivalente en la que encontramos un diccionario general, formado por artículos de otros proyectos lexicográficos como el diccionario Espasa-Calpe, un diccionario de sinónimos y antónimos, una gramática del español y, finalmente, un conjunto de diccionarios bilingües de distintas lenguas. Si bien los dos tienen una finalidad descodificadora (conocer el significado de la palabra) y, en menor medida, codificadora (usar la palabra), ninguno de los dos es un diccionario didáctico. En este sentido, conviene señalar que los profesores aconsejan el uso de diccionarios generales, pero no mencionan los diccionarios escolares, que tienen como destinatario a un estudiante no universitario que quiere mejorar el uso de su lengua materna (Azorín Fernández, 2000). Este tipo de obras son relevantes en la ESO, ya que, si el usuario tiene alguna carencia léxica, precisa la 
explicación de algún dato que un hablante competente en español puede dar por sabido.

Asimismo, es importante la realización de actividades pautadas por el docente a lo largo de las etapas escolares (Hernández Hernández, 1991; Prado Aragonés, 1996; Maldonado González, 1998, 2003; Martín García, 1999; Martínez Ezquerro, 2001, entre otros). Durante los primeros años de la ESO, el conocimiento de la obra lexicográfica va a servir, fundamentalmente, para tratar la morfología flexiva, ya que es el momento en el que surgen más dudas morfológicas sobre los paradigmas flexivos (Candalija Reina y Marimón Llorca, 2001), además de ciertas cuestiones derivadas del análisis morfológico como la categoría gramatical, las irregularidades del verbo o la flexión verbal. Posteriormente, el diccionario puede favorecer el aprendizaje de la morfología léxica mediante las relaciones en las familias léxicas y la información contenida en los artículos lexicográficos de los afijos y de las propias palabras derivadas incluidas en el listado alfabético.

Sin embargo, como muestran los datos de la encuesta, el diccionario no suele ser un recurso para enseñar morfología, a pesar de que el docente lo use para otros fines. Si bien la mayoría de los profesores opina que el empleo del diccionario facilita la adquisición de los conceptos morfológicos que se plantean en el párrafo anterior (morfología flexiva, categorías gramaticales y morfología léxica), son menos los que aúnan morfología y lexicografía en el aula.

No obstante, los resultados de la encuesta indican que aquellos docentes que utilizan el diccionario para enseñar morfología optan por realizar las actividades del libro de texto o por elaborar sus propias actividades. Por un lado, el uso del manual es el reflejo de que cada vez es más habitual encontrar apartados en los libros de texto donde aparecen exposiciones sobre los diccionarios y actividades relacionadas con su manejo (Martínez Montoro, 2006, pp. 209-222 y Rodríguez Márquez, 2017, pp. 94-96). Por otro lado, este dato nos lleva a pensar una cuestión: ¿los once docentes que crean ejercicios son mayoritariamente los que han indicado que poseen formación lexicográfica? Después de haber cruzado los datos, observamos que 
cinco de los once encuestados cumplen los requisitos de una formación específica. Es decir, los conocimientos sobre lexicografía influyen en la elaboración de actividades relacionadas con el manejo del diccionario y la morfología.

En conclusión, en este apartado hemos expuesto los datos de la encuesta realizada de forma anónima a treinta profesores de Lengua Castellana y Literatura en el contexto de la ESO. Hemos observado que las características personales de los participantes, tanto su nivel de formación y sus conocimientos en lexicografía como su hábito en la consulta de diccionarios, influyen en la percepción que tienen los docentes en el uso del diccionario en el aula. Asimismo, los datos indican que cada vez son más los profesores que incluyen el diccionario en el aula. Sin embargo, no suelen apoyarse en este tipo de materiales para tratar cuestiones morfológicas, aunque son conscientes de los beneficios que puede aportar su uso. Además de las cuestiones linguísticas, los docentes admiten que la consulta del diccionario fomenta la autonomía en el proceso de enseñanza-aprendizaje del estudiante.

Después de haber analizado en este apartado los datos de la encuesta, es el momento de plantearnos cómo se puede usar el diccionario para explicar morfología en el aula de ESO.

\section{3.- La enseñanza de la morfología a través del diccionario 3.1.- La morfología en el diccionario}

Según lo que hemos comentado en el apartado anterior, son pocos los docentes que deciden utilizar el diccionario para enseñar cuestiones morfológicas en el aula. Es por ello por lo que vamos a plantear unas actividades que se podrían seguir para enseñar morfología con el diccionario. Asimismo, es necesario aclarar que no consideramos que el uso de obras lexicográficas deba ser una práctica constante en el aula ni debe ser obligatorio. Lo que pretendemos es que exista la posibilidad de emplear este recurso en clase para que el estudiante pueda acudir a él cuando tenga una duda morfológica, con el fin de fomentar el aprendizaje autónomo y la reflexión lingüística. 
Respecto al modelo didáctico que asumamos, este ha de tener en cuenta los siguientes aspectos (Bosque Muñoz, 2015). Primero, debe fomentar la reflexión metalinguiística a través de una teoría sencilla y una terminología cercana al estudiante (Querol Bataller, 2015). En las primeras etapas de la educación, las cuestiones terminológicas no pueden suponer un obstáculo para complicar la comprensión de la estructura de la lengua. Segundo, la enseñanza de la gramática debe basarse en un aprendizaje significativo para que el estudiante actúe de manera activa en el descubrimiento de la lengua. Tercero, la relación entre el signo linguístico y su significado debe evidenciarse a partir de construcciones sencillas que faciliten su comprensión. Por último, el modelo tiene que permitir la ampliación de conocimientos de manera paulatina. Es decir, debe haber una progresión de contenidos para que el estudiante pueda afianzar la información adquirida y pueda relacionar los datos nuevos con los vistos previamente.

Teniendo presente las ideas anteriores, presentaremos unas actividades a partir del Diccionario de uso del español actual-Clave (DUEA-CLAVE) de la editorial SM. ${ }^{3}$ La elección de este diccionario, disponible en papel y en línea, se debe a que permite hacer búsquedas tanto de la palabra como de los prefijos o los sufijos que la componen. Además, al buscar una determinada palabra, pueden aparecer listadas todas las de la familia léxica que la contienen, de modo que el usuario seleccionará aquella que más se ajuste a su necesidad comunicativa.

Por ejemplo, al escribir en el buscador el término surf y marcar la opción "Contiene", el Diccionario DUEA-CLAVE muestra todas las palabras que contienen el lema surf en la macroestructura.

3 Seguiremos la versión en línea del diccionario http://clave.smdiccionarios.com/app.php. Consultado durante los meses de septiembre 2020 y abril 2021. 


\section{Figura 5}

Entrada de kitesurf en el DUEA-CLAVE

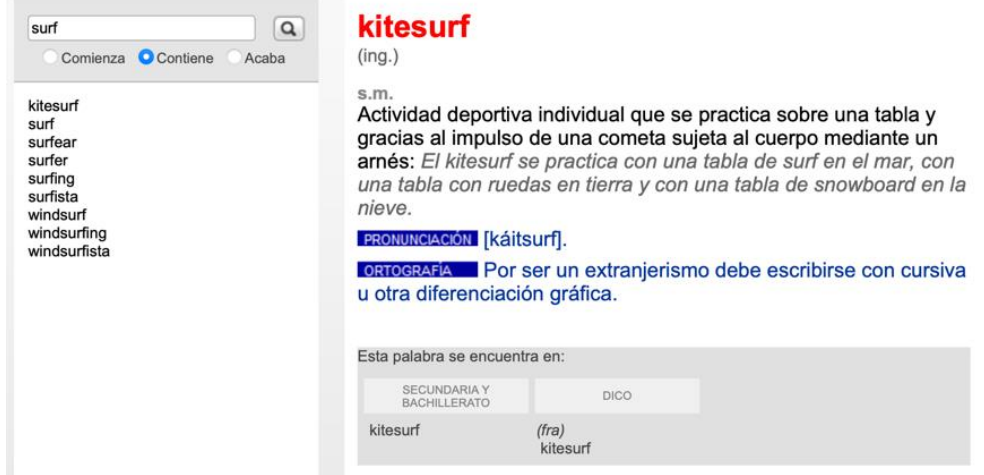

Fuente: DUEA-CLAVE, s.f.

De esta manera, se puede tratar la morfología léxica a partir de las familias. Las palabras derivadas que forman parte de la misma familia léxica crean un esquema homogéneo, ya que comparten el lexema, como en el caso de surf, surfear, surfista y kitesurf. Aunque el DUEA-CLAVE indique las palabras que contienen el lema surf, en ningún apartado del artículo lexicográfico se muestra el proceso de formación de la palabra. Por ejemplo, surfear es una palabra derivada; mientras que kitesurf es compuesta.

Sin embargo, si buscamos el lema enfermedad con la opción "Contiene", vemos que el DUEA-CLAVE solo selecciona las palabras que coinciden formalmente con la que se escribe en el buscador. En el apartado de búsqueda, solo aparece el término enfermedad; pero no enfermo, enfermar o enfermería. Por lo tanto, esta función permite detectar familias de palabras solo cuando existe una coincidencia exacta. Para solucionar este problema, el usuario tiene que hacer la búsqueda a través de la raíz del término (enferm-, por ejemplo), lo que conlleva saber descomponer previamente la palabra deseada.

Como vemos en las unidades léxicas elegidas, las relaciones entre las familias pueden ser diversas, por lo que el nivel de dificultad será diferente según los términos que se elijan. Por un lado, los derivados de surf son más transparentes desde el punto de vista formal que la familia de escuela. Por otro lado, los afijos suelen ser 
polisémicos, por lo que los significados deben aparecer codificados en las entradas de los afijos en los diccionarios. Así, el sufijo -ero puede crear sustantivos denominales con el significado de 'persona que trabaja con o en N' (relojero, jardinero), 'lugar donde hay N' (basurero), pero también puede dar lugar a adjetivos denominales de relación (sector algodonero) o de propiedad (aventurero), como se refleja en la entrada de niñero, con dos significados: el adjetivo calificativo (acepción 1) y el sustantivo de profesión (acepción 2).

\section{Figura 6}

Entrada de niñero en el DUEA-CLAVE

niñero, ra ni.ñe·ro, ra

adj.

1 Que disfruta estando con niños.

s.

2 Persona empleada en una casa para cuidar a los niños.

SEMAMTICA En la acepción 2, el femenino es sinónimo de chacha.

USo En la acepción 2, es innecesario el uso del anglicismo nurse.

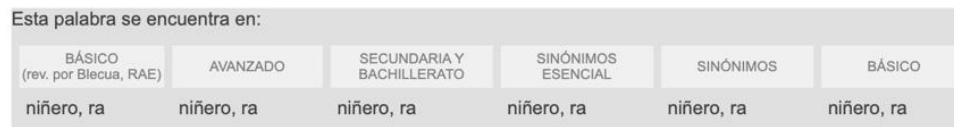

Fuente: DUEA-CLAVE, s.f.

Asimismo, si el usuario quiere conocer qué palabras contienen el sufijo -ero, puede utilizar la opción "Acaba" y le aparecerán en el buscador todos los términos que terminen en -ero. En cambio, este tendrá que descubrir cuáles son las palabras derivadas con -ero (abacero o abaniquero) y cuáles son los términos simples que acaben en -ero (acero o agujero). Además, sucede una situación similar con las búsquedas hechas con "Comienza".

Por último, el DUEA-CLAVE tiene la opción de buscar cuál es el significado de prefijos y sufijos, a través del uso de "Comienza" y "Acaba", respectivamente. Como vemos en el siguiente ejemplo: 
Figura 7

Entrada de -ero en el DUEA-CLAVE

\section{-ero, -era}

1 Sufijo que indica oficio o actividad: librero, ingeniera.

2 Sufijo que indica relación: verbenero, algodonera.

3 Sufijo que indica cualidad: pamplinero, embustera.

ETIMOLOGIA Del latín -arius.

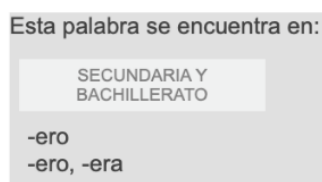

Fuente: DUEA-CLAVE, s.f.

A continuación, presentamos una serie de actividades basadas en lo que acabamos de indicar para tratar la morfología léxica.

\section{2.- Modelo de enseñanza de la morfología léxica}

Como indica Gallego Bartolomé (2016) las actividades gramaticales que se encuentran en los libros de texto de la ESO buscan contabilizar los aciertos y fallos de los estudiantes. Sin embargo, son pocos los casos que fomentar la reflexión a través de la justificación o explicación del fenómeno. En este apartado se presenta una muestra de actividades basadas en los pares mínimos y en la reflexión lingüística. Hemos dividido estas actividades según las opciones de búsqueda que presenta el DUEA-CLAVE ("Comienza", "Contiene" y "Acaba").

Primero, la opción "Comienza" se utiliza para reflexionar sobre la prefijación. En (1), se muestran los adjetivos preconstitucional y predorsal con el objetivo de que el estudiante analice el significado del prefijo pre-.

(1) a. Es un acuerdo preconstitucional

b. Es un dolor predorsal 
En este par mínimo y a través del botón "Comienza", se busca el prefijo pre- en el DUEA-CLAVE para comprobar si preconstitucional y predorsal tienen algún significado común. El prefijo aparece con dos acepciones: una con valor temporal y otra con valor locativo. El estudiante tendrá que justificar cuál se corresponde a cada uno de los términos.

Otra posibilidad es concienciar al estudiante de que existen palabras en las que es posible identificar formalmente un afijo pero sin significado, dado que la palabra está lexicalizada. En (2), se muestran dos verbos que llevan pre-. El estudiante tendrá que determinar si en los dos ejemplos pre- es un prefijo y si los dos verbos comparten un significado común.

\section{a. Preparar el desastre}

b. Predecir el desastre

En segundo lugar, con la opción "Contiene" es posible encontrar palabras de una familia léxica para reflexionar sobre las nociones de raíz y de familia léxica, a la vez que el estudiante visualiza las relaciones entre el léxico.

Busca en el diccionario DUEA-Clave la palabra pesca y otras de su misma familia léxica. ¿Cuál es la raíz de esta familia léxica? ¿Pertenecen a esta familia palabras como pescozón o pescuezo? Intenta explicar por qué. Ahora intenta definir la noción de raíz.

En tercer lugar, con la opción "Acaba" hemos elegido pares mínimos para que el estudiante sea capaz de determinar la categoría gramatical de las palabras con un significado relacionado. En (3), se muestran las palabras surfear, surf y surfista en un mismo contexto.
a. Surfear es peligroso
b. El surf es peligroso
c. El surfista es peligroso

El estudiante debe advertir que los sufijos tienen la capacidad de modificar el significado y la categoría gramatical de la raíz. Con la 
opción "Acaba", es posible buscar los sufijos -ear e -ista, así como otras palabras que llevan estos sufijos. De este modo, el estudiante podrá identificar los constituyentes, relacionar las palabras y reconocer las categorías gramaticales según el sufijo.

Por último, en (4), se trabaja el concepto de alomorfía.

(4) Fíjate en las siguientes dos series de palabras:

a. jugador, nadador y decorador

b. conductor, traductor y productor

¿Hay alguna relación entre las palabras de los dos grupos? ¿Qué significan? ¿Qué elemento aporta este significado? Puedes usar el diccionario DUEA-CLAVE para ayudarte en tu explicación. Ahora añade a la lista anterior más ejemplos.

Con la opción "Acaba” del DUEA-CLAVE, el estudiante puede encontrar más palabras terminadas en -dor y -tor, a la vez que entiende la relación entre las dos formas del sufijo. De este modo, es el estudiante el que selecciona las palabras de una lista teniendo en cuenta que son palabras derivadas y que comparten el mismo significado del sufijo.

\section{Conclusiones}

En este artículo hemos analizado la relación entre lexicografía y morfología en el ámbito escolar a través de un estudio preliminar. Mediante los datos resultantes de la encuesta realizada a profesores de Lengua Castellana y Literatura hemos podido responder a los objetivos que plateamos en la introducción. Por un lado, hemos apreciado que el uso del diccionario en la ESO está cada vez más asentado y, por otro, que la inclusión de obras lexicográficas para explicar morfología es escasa.

Centrándonos en el uso del diccionario monolingüe en el ámbito escolar, podemos afirmar que es un tema recurrente en los trabajos sobre lexicografía didáctica desde finales del siglo pasado. No obstante, aunque los profesores han mostrado que la utilización del diccionario 
está cada vez más extendida, sería conveniente fomentar la formación de los docentes en lexicografía a través de programas concretos adaptados al contexto educativo.

Asimismo, la morfología es uno de los campos lingüísticos comunes en la ESO. Durante esta etapa es primordial que los estudiantes entiendan la estructura interna de las palabras complejas, de modo que fácilmente puedan deducir su significado y aprendan otras propiedades gramaticales asociadas a los constituyentes morfológicos. Sin embargo, son pocos los profesores que optan por incluir el uso del diccionario a la hora de explicar morfología. Para evitar esta situación, es conveniente adoptar líneas didácticas que aúnen el estudio de la morfología y el uso del diccionario, atendiendo a las tres finalidades que debe perseguir la enseñanza de un idioma: comunicar, reflexionar sobre la propia lengua y crear referentes culturales (Dolz Mestre, Gagnon y Mosquera Roa, 2009, p. 125).

En este artículo hemos mostrado, a modo de ejemplo, algunas actividades de reflexión sobre las palabras derivadas con ayuda del material lexicográfico. En este sentido, el Diccionario DUEA-Clave en línea permite trabajar el léxico de una manera diferente, gracias a las opciones "Comienza", "Contiene" y "Acaba". Con estos recursos se fomenta la comprensión del léxico y, a través de la reflexión, se facilita la ampliación del vocabulario y la consolidación de las relaciones léxicas. 


\section{Referencias bibliográficas}

Alvar Ezquerra, M. (1993). Lexicografía descriptiva. Barcelona: Biblograf.

Alvar Ezquerra, M. (2003). La enseñanza del léxico y el uso del diccionario. Madrid: Arco/Libros.

Azorín Fernández, D. (2000). Los diccionarios didácticos del español desde la perspectiva de sus destinatarios. ELUA. Estudios De Lingüística, 14, 19-44. doi:10.14198/ELUA2000.14.02

Battaner Arias, P., y Torner Castells, S. (s.f.) Diccionario de aprendizaje del español como lengua extranjera. Recuperado de http://www.iula.upf.edu/rec/daele/

Bosque Muñoz, I. (2015). "Nuevas reflexiones sobre la enseñanza de la gramática: actitudes frente a contenidos". II Jornadas GrOC. Barcelona: UAB. 5 de febrero de 2015. Recuperado de https://www.dropbox.com/s/8g6ujy22ihuhrct/_Bosque\%20GrOC\%2020 15.pdf?dl=0

Candalija Reina, J. A., y Marimón Llorca, M. C. (2001). La información gramatical en los diccionarios escolares: reflexiones a propósito de una encuesta sobre el uso del diccionario. En Ruhstaller, S., Prado Aragonés, J. (Eds.), Tendencias en la investigación lexicográfica del español. El diccionario como objeto de estudio lingüístico y didáctico, (pp. 311-321). Huelva: Universidad de Huelva.

Decreto 48/2015, de 14 de mayo, por el que se establece para la Comunidad de Madrid el currículo de la Educación Secundaria Obligatoria. Boletín Oficial de la Comunidad de Madrid, 118, de 20 de mayo de 2015, 10-118. Recuperado de https://www.bocm.es/boletin/CM_Orden_BOCM/2015/05/20/BOCM20150520-1.PDF

Cassany Comas, D., Luna Sanjuan, M., y Sanz Pinyol, G. (1994). Enseñar lengua. Barcelona: Graó. Recuperado de http://lenguaydidactica.weebly.com/uploads/9/6/4/6/9646574/cassany, d._luna,_m._sanz,_g._-_ensenar_lengua.pdf

De Schryver, G. (2003). Lexicographers' dreams in the electronic-dictionary age. International Journal of Lexicography, 16(2), 143-199. doi:10.1093/ijl/16.2.143 
Dolz Mestre, J., Gagnon, R., y Mosquera Roa, S. (2009). La didáctica de las lenguas: Una disciplina en proceso de construcción. Didáctica. Lengua y Literatura, 21, 117-141. Recuperado de https://revistas.ucm.es/index.php/DIDA/article/view/DIDA0909110117 $\mathrm{A} / 18815$

Dubois, J., y Dubois, C. (1971). Introduction à la lexicographie: Le dictionnaire. París: Larousse.

Gallego Bartolomé, A. J. (2016). Sobre los ejercicios de "reflexión gramatical": malentendidos, ventajas metodológicas y aplicaciones didácticas. Revista Española de Lingüística, 46, 145-158. Recuperado de https://dialnet.unirioja.es/descarga/articulo/6315991.pdf

Graves, M. F. (2006). The vocabulary book: Learning and instruction. Nueva York: Teachers College Press, Columbia University.

Hernández Hernández, H. (1989). Los diccionarios de orientación escolar: Contribución al estudio de la lexicografía monolingüe española. Tübingen: Max Niemeyer Verlag. Recuperado de http://riull.ull.es/xmlui/handle/915/24492

Hernández Hernández, H. (1991). De la teoría lexicográfica al uso del diccionario: el diccionario en el aula. En S. Montesa Peydró y A. Garrido Moraga (Coords.), El español como lengua extranjera: de la teoría al aula. Actas Del III Congreso Nacional ASELE (189-200). Málaga. Recuperado de https://dialnet.unirioja.es/descarga/articulo/1959462.pdf

Hernández Hernández, H. (1996). La lexicografía didáctica: Los diccionarios escolares del español en el último cuarto de siglo. Cuadernos Cervantes, 11, 24-36.

Hernández Hernández, H. (1998). La lexicografía didáctica del español: Aspectos históricos y críticos. En M. T. Fuentes Morán, y W. Reinhold (Eds.), Lexicografías iberorrománicas: Problemas, propuestas y proyectos, (49-79). Frankfurt am Main: Vervuert Verlag.

Hess Zimmermann, K. (2019). Pensar sobre la morfología de las palabras: Un proyecto didáctico para el desarrollo de vocabulario en la escuela secundaria. Revista iberoamericana de evaluación educativa, 12 (2), 193-215. doi: 10.15366/riee2019.12.1.002.010

Kieffer, M. J., y Lesaux, N. K. (2012). Effects of academic language instruction on relational and syntactic aspects of morphological awareness for sixth graders from linguistically diverse 
backgrounds. The Elementary School Journal, 3(112), 519-545. doi: $10.1086 / 663299$

Larsen, J. A., y Nippold, M. A. (2007). Morphological analysis in school-age children: Dynamic assessment of a word learning strategy. Language, Speech and Hearing Services in Schools, 38, 201212.

Recuperado

de https://www.researchgate.net/publication/6213247_Morphological_Ana lysis_in_School-

Age_Children_Dynamic_Assessment_of_a_Word_Learning_Strategy

Levie, R., Ben-Zvi, G., y Ravid, D. (2016). Morpho-lexical development in language impaired and typically developing Hebrewspeaking children from two SES backgrounds. Reading and Writing, 5(30), 1035-1064. doi: 10.1007/s11145-016-9711-3

Maldonado González, C. (s.f.) Diccionario clave de uso del español. Recuperado de http://clave.smdiccionarios.com/app.php

Maldonado González, C. (2003). La lexicografía didáctica monolingüe en español. En M. A. Martín Zorraquino y J. L. Aliaga Jiménez (Eds.). La lexicografía hispánica ante el siglo XXI. Balance y perspectivas (pp.129-150). Zaragoza: Sansueña Industrias Gráficas.

Maldonado González, C. (1998). El uso del diccionario en el aula. Madrid: Arco/Libros.

Martín García, J. (1999). El diccionario en la enseñanza del español. Madrid: Arco/Libros.

Martín García, J. (2015). La codificación de la información morfológica en los diccionarios de ELE. Relaciones Morfológicas y Diccionario, 36, 35-48.

Martín Vegas, R. A. (2018). Modelos de aprendizaje léxico basados en la morfología derivativa. Rilce, 1(34), 262-285. doi: 10.15581/008.34.1.262-85

Martínez Ezquerro, A. (2001). Competencia curricular y diccionario. Contextos Educativos, 4, 315-328. Recuperado de https://publicaciones.unirioja.es/ojs/index.php/contextos/article/view/49 9/463

Martínez Fraile, S. (2015). Els conceptes morfològics bàsic en l'ensenyament secundari. Tejuelo: Didáctica de Lengua y la Literatura. Educación, $22(1)$ 191-214. Recuperado de http://hdl.handle.net/10662/5048 
Martínez Montoro, J. (2006). El diccionario ideológico de Julio Casares: notas para su enseñanza. En J. A. Moya Corral y M. Sosinski (Coords.), Lexicografía y enseñanza de la lengua española. Actas de las XI Jornadas de Enseñanza de Lengua Española (209-222). Granada: Editorial Universidad de Granada.

Matilla Ocaña, C. (2006). "La piel de la lengua" o enseñanza y aprendizaje del léxico. En J. A. Moya Corral y M. Sosinski (Coords.), Lexicografía y enseñanza de la lengua española. Actas de las XI Jornadas de Enseñanza de Lengua Española (223-238). Granada: Editorial Universidad de Granada.

Ministerio de Educación y Formación Profesional (2015). Competencias Clave.

Recuperado de https://www.educacionyfp.gob.es/educacion/mc/lomce/curriculo/compe tencias-clave/competencias-clave.html

Nation, P. (1990). Teaching and learning vocabulary. New York: Newbury House.

Nippold, M. A. (2006). Later language development: Schoolage, adolescents, and young adults. Encyclopedia of language \& linguistics (pp. 368-373). Oregon: University of Oregon. doi: 10.1016/B0-08-044854-2/00852-X

Prado Aragonés, J. (1996). Usos creativos del diccionario en el aula. Cuadernos Cervantes, 11, 38-45.

Prado Aragonés, J. (2005). El uso del diccionario para la enseñanza de la lengua: Consideraciones metodológicas. Káñina, 29, 19-28. Recuperado de https://revistas.ucr.ac.cr/index.php/kanina/article/view/4653/4467

Querol Bataller, M. (2015). Propuestas para "llevar la gramática a los alumnos". Tejuelo: Didáctica de Lengua y la Literatura. Educación, 22(1), 141-167. Recuperado de: http://hdl.handle.net/10662/4927

RAE, y ASALE (2009). Nueva gramática de la lengua española. Madrid: Espasa.

Real Decreto 1105/2014, de 26 de diciembre, por el que se establece el currículo básico de la Educación Secundaria Obligatoria y del Bachillerato. Boletín Oficial del Estado, 3, de 3 de enero de 2015, 169-546.

Recuperado de https://www.boe.es/boe/dias/2015/01/03/pdfs/BOE-A-2015-37.pdf 
Rodríguez Márquez, T. I. (2017). La lexicografía y el diccionario como herramienta en la enseñanza secundaria: Unidades de trabajo. Barcelona: Universitat Autònoma de Barcelona.

Sánchez García, D. (2015). Teaching learning and communicative strategies. En L. R. Buckingham (Ed.), EFL teaching and learning I (pp. 199-220). Madrid: CEF.

Sánchez Muñoz, T. (2003). Los diccionarios escolares a comienzos del siglo XXI. Revista de lexicografía, 9, 175-188. doi: 10.17979/rlex.2003.9.0.5581

Saragossá Alba, A. (2015). La morfologia i els seus conceptes en l'ensenyament secundari: tractaments habituals. Tejuelo: Didáctica de Lengua y la Literatura. Educación, 22(1), 168-190. Recuperado de http://hdl.handle.net/10662/5098 